\title{
The dynamical evolution of fractal star clusters: The survival of substructure
}

\author{
S. P. Goodwin and A. P. Whitworth \\ Dept. of Physics \& Astronomy, Cardiff University, 5 The Parade, Cardiff, CF24 3YB, UK \\ Received 16 April 2003 / Accepted 25 September 2003

\begin{abstract}
We simulate the dynamics of fractal star clusters, in order to investigate the evolution of substructure in recently formed clusters. The velocity dispersion is found to be the key parameter determining the survival of substructure. In clusters with a low initial velocity dispersion, the ensuing collapse of the cluster tends to erase substructure, although some substructure may persist beyond the collapse phase. In clusters with virial ratios of 0.5 or higher, initial density substructure survives for several crossing times, in virtually all cases. Even an initially homogeneous cluster can develop substructure, if it is born with coherent velocity dispersion.

These results suggest that the simple initial conditions used for many sophisticated $N$-body simulations could be missing a very important and dramatic phase of star cluster evolution.
\end{abstract}

Key words. stellar dynamics - open clusters and associations: general

\section{Introduction}

It appears that most stars - possibly all stars - form in clusters. Their dynamical evolution is thus of great interest. In recent years, codes such as NBODY6 (Aarseth 2000), which include detailed stellar evolution and mass loss, binary evolution and mass transfer, have made possible a new generation of "kitchen sink" simulations (e.g. Kroupa et al. 2001; Hurley et al. 2001; Portegies Zwart et al. 2001). However, the initial conditions for these simulations have often been very simple, for example Plummer models, in stark contrast with the great detail invoked in modelling the subsequent evolution.

Observations of star clusters, on the other hand, suggest that the initial conditions for star formation are highly clumpy and structured, both in the distribution of the molecular gas from which stars are about to form (e.g. Williams 1999 and references therein), and in the distribution of newly-formed stars (e.g. Bate et al. 1998; Gladwin et al. 1999).

Aarseth \& Hills (1972) were the first to investigate the evolution of collapsing star clusters with substructure. Their simulations were limited by the available computer power to clusters of 120 stars. They found that subclustering was destroyed on a free-fall timescale. Later Goodwin (1998) investigated an initially virialised cluster with density substructure and a larger number of stars. He found that most of the initial substructure was erased within a few crossing times.

In this paper we investigate the evolution of initially fractal star clusters to see how long substructure can survive.

Send offprint requests to: $\mathrm{S}$. P. Goodwin, e-mail: Simon.Goodwin@astro.cf.ac.uk
Our models include star clusters with large velocity dispersions, as would be expected in clusters shortly after they expel their residual gas (cf. Goodwin 1997). In addition, we investigate fractal clusters in which the density substructure is correlated with coherent velocity dispersion, as would be expected in clusters where sub-clusters form from distinct molecular cores.

We are not suggesting that star clusters are necessarily fractal. The range of scales over which young star clusters exhibit substructure is usually very small, often less than an order of magnitude, so the notion of a fractal cannot be applied rigorously. Nonetheless, fractals provide a simple, one-parameter description of clumpiness, and this is why we are using them.

\section{2. $\mathrm{N}$-body method and initial conditions}

We conduct our $N$-body simulations on a GRAPE- 5 board, which allows for very rapid solution of the $N$-body gravitational problem (Kawai et al. 2000). We use a simple direct first order $N$-body integrator, as the speed of the GRAPE- 5 board allows the timestep to be set sufficiently small that over the course of a simulations the total energy of the system never changes by more than 0.01 per cent, and usually by significantly less than this. A small softening length is used (generally of order $10^{-5}$ in code units).

The presence of binaries in a cluster will effect the dynamical evolution of the system by altering impact parameters. However, tests using different softening lengths show the results to be independent of the softenning length, even when it is significantly larger than our canonical $10^{-5}$. This is due to the relaxation being primerally violent relaxation, rather than encounter-driven two-body relaxation. 
Fractal distributions are generated by defining an $u r$-cube with side 2, and placing an $u r$-parent at the centre of the $u r$-cube. Next, the $u r$-cube is divided into $N_{\text {div }}^{3}$ equal subcubes, and a child is placed at the centre of each sub-cube (the first generation). Normally we use $N_{\text {div }}=2$, in which case there are 8 sub-cubes and 8 first-generation children. The probability that a child matures to become a parent in its own right is $N_{\text {div }}^{(D-3)}$, where $D$ is the fractal dimension; for lower $D$, the probability that a child matures to become a parent is lower. Children that do not mature are deleted, along with the $u r$-parent. A little noise is then added to the positions of the remaining children, to avoid an obviously gridded structure, and they become the parents of the next generation, each one spawning $N_{\text {div }}^{3}$ children (the second generation) at the centres of $N_{\text {div }}^{3}$ equal-volume sub-sub-cubes, and each secondgeneration child having a probability $N_{\text {div }}^{(D-3)}$ of maturing to become a parent. This process is repeated recursively until there is a sufficiently large generation that, even after pruning to impose a spherical envelope of radius 1 within the $u r$-cube, there are more children than the required number of stars. Children are then culled randomly until the required number is left, and the survivng children are identified with the stars of the cluster.

We explore a range of models with $N_{\text {tot }}=1000$ and $N_{\text {tot }}=10000$ stars. The fractal dimensions which we investigate are $D=1.6,2.0,2.6$, and 3.0, since these all correspond to $2^{D}$ (the mean number of maturing children) being close to an integer. This reduces the likelihood of departures from the specified fractal dimension, because in our algorithm for constructing an initial cluster the number of maturing children born to each parent should be an integer.

Our simulations begin with a random velocity dispersion, which is either incoherent or coherent. For an incoherent velocity dispersion, each particle is given random Cartesian velocity components from a Gaussian distribution, and these velocities are then scaled so that the virial ratio $\alpha$ has the prescribed value. $\alpha$ is defined as the ratio of the total kinetic energy to the magnitude of the gravitational potential energy. $\alpha=0.1$ corresponds to a cluster which immediately collapses. $\alpha=0.5$ corresponds to virial equilibrium. $\alpha=0.75$ corresponds to a super-virial cluster (i.e. one which has just expelled its residual gas).

For a coherent velocity dispersion, each star inherits most of its velocity from its family tree. We first calculate, for each mature child of each generation, how many stars are descended from it, and this gives the child's mass. Next, starting with the children of the first generation, we give them random velocities relative to the $u r$-parent, so that they form a virialised cluster. We treat the children of each subsequent family in the same way, giving them random velocities relative to the parent, so that they form a virialised sub-cluster, but in addition they acquire the velocity of the parent. This process is repeated recursively, and hence the stars of the final generation inherit random velocities from all their antecedents, giving a coherent velocity dispersion. Finally, the velocities are scaled so that the virial ratio $\alpha$ has the prescribed value.

We believe that the most realistic of the initial velocity dispersions we use is the coherent, super-virial one ( $\alpha=0.75)$. Our simulations do not include a gaseous component, but we assume that the residual gas has been expelled from the cluster very rapidly and recently. If the stars have previously been in virial equilibrium with the residual gas, the virial ratio of the purely stellar component is super-virial. It also seems likely that the velocities of the stars will be correlated locally, on the assumption that each sub-cluster of stars has formed from a single molecular core.

\section{Measures of clumpiness}

The primary aim of this paper is to investigate how rapidly initial density substructure and associated velocity coherence are destroyed, and the typical timescale before a cluster can be described as smooth. In order to do this we require a robust method for measuring the clumpiness of a cluster.

One method would be to determine the evolution of the fractal dimension of the cluster. However, this method has drawbacks, because it is difficult to measure the fractal dimension of a distribution with a strong overall radial density gradient. Clusters rapidly acquire a core-halo structure, in which the mean separation between stars decreases radially by a significant factor. To determine the fractal box-dimension of a cluster, a grid of cells is overlaid on the cluster, and the number of cells containing at least one star is counted. This is repeated, starting with a coarse grid of large cells, and proceeding to ever finer grids of smaller cells. The fractal box-dimension is then given by the slope of a plot of the log of the number of occupied cells against the log of the inverse cell size. This slope turns over at the point where the grid becomes saturated, i.e. where the number of occupied cells is equal to the number of stars; further reductions of cell size then make no difference to cell occupancy. However, in the presence of a density gradient, saturation of the halo occurs before saturation of the core, producing a complicated relationship that is difficult to interpret. It might be possible to compensate for this, if the overall radial density distribution is known a priori, but in general it is not.

The simple method we use to measure clumpiness is to accord each star a local density, given by $5 m / V_{5}$, where $m$ is the stellar mass and $V_{5}$ is the spherical volume bounded by the fifth nearest star. Next we overlay spherical shells centred on the centre of mass of the ten densest stars, and work out the average density in each shell. Substructure is then visible as stars (or groups of stars) within a shell having density significantly higher than the average density for that shell. As a measure of the level of clumpiness, we use two numbers, $F_{20}$ and $F_{50} . F_{20}\left(F_{50}\right)$ measures the fraction of shells in which more than 20\% (50\%) of the stars have more than 5 times the average density. A high level of clumpiness is reflected in large values of $F_{20}$ and $F_{50}$. We tested more complicated, kernel weighted density estimates (such as those used in SPH simulations), but found no significant difference in the results.

$F_{20}$ and $F_{50}$ are not greatly affected by small number statistics. Tests performed with clusters of 1000 stars show that $F_{50}<F_{20} \lesssim 0.1$ for various clusters which should not have significant clumpiness, viz. a randomly distributed uniformdensity cluster, a randomly distributed Plummer cluster, and a $D=3$ fractal cluster. In contrast, a $D=1.6$ fractal cluster, which should be very clumpy, has $F_{50} \sim F_{20} \sim 1$. 

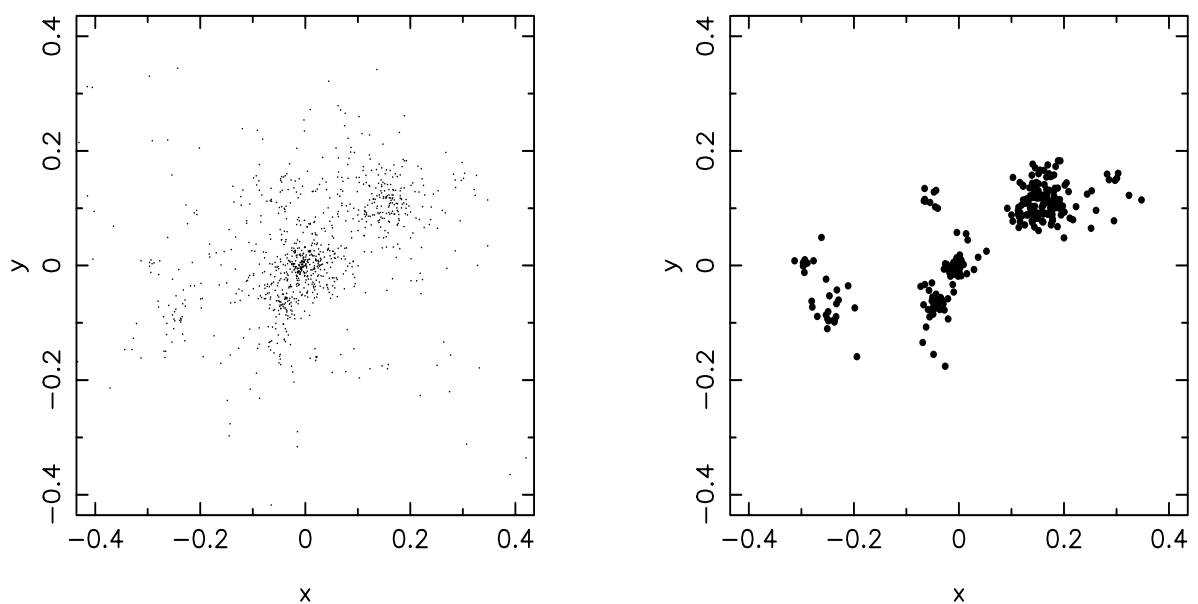

Fig. 1. Left: a clumpy distribution of stars. Right: the stars selected by our clumpiness measure as being significantly overdense. This distribution has high $F_{20}$ and $F_{50}$ which indicate that it is significantly clumpy (which the eye confirms).
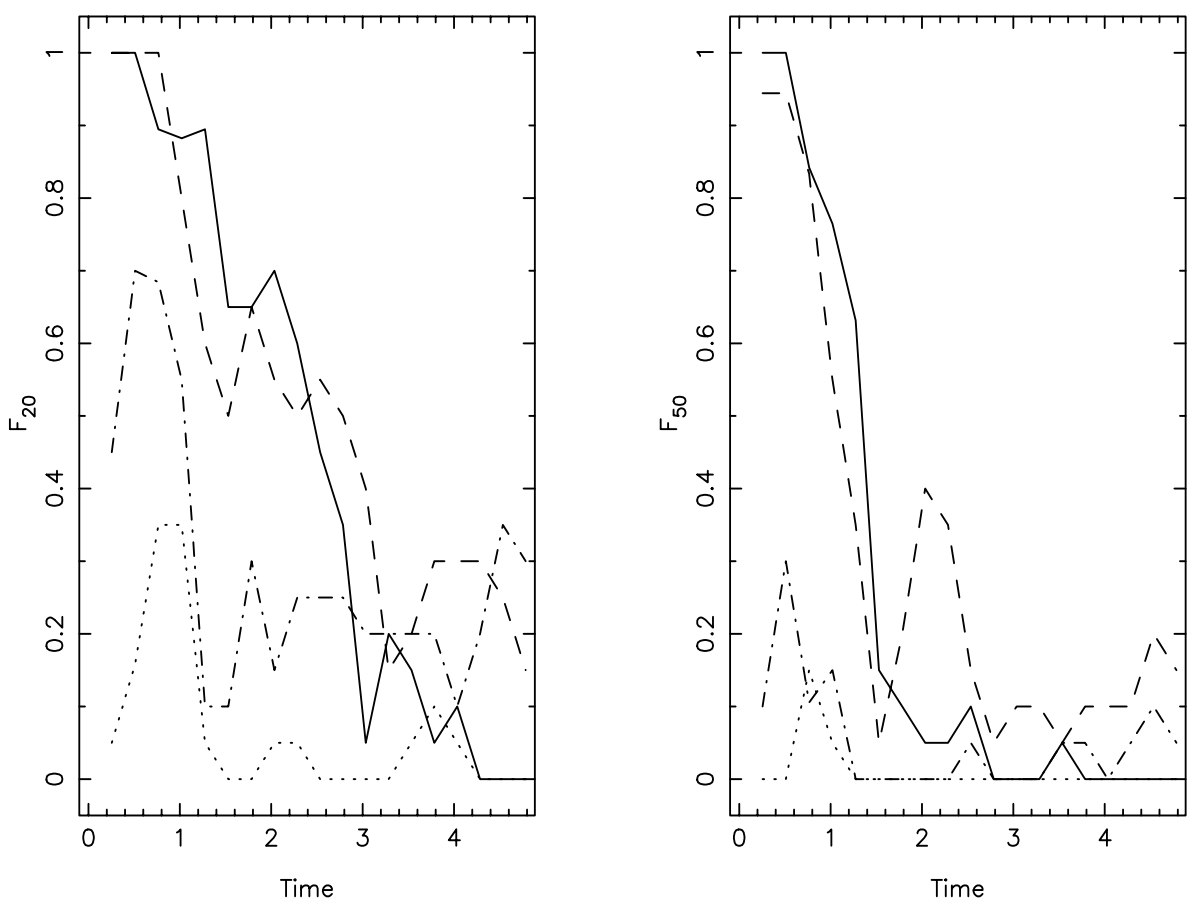

Fig. 2. The evolution of the $F_{20}$ and $F_{50}$ measures of clumpiness for clusters with 1000 particles, initial virial ratio 0.1 , coherent velocities and initial $D$ of 1.6 (solid lines), 2 (dashed lines), 2.6 (dot-dashed lines) and 3 (dotted lines).

We conclude that $F_{20}$ and $F_{50}$ are useful measures of clumpiness. Figure 1 shows a fractal distribution with obvious substructure, and the large points show which stars have been selected by this method as being 5 times the average density. They coincide well with the substructure which the human eye identifies.

\section{The evolution of fractal clusters}

We now use $F_{20}$ and $F_{50}$ to investigate the evolution and erasure of substructure from an initially fractal cluster.

\subsection{Collapsing clusters}

Figure 2 shows typical results for the evolution of $F_{20}$ and $F_{50}$ for clusters having initial virial ratio $\alpha=0.1$ (such that they will collapse) and initial fractal dimensions $D=1.6,2.0,2.6$, and 3 (decreasing levels of initial substructure). In all of these cases the initial velocity dispersion is coherent.

Time is given in $N$-body units, such that $G=M=R=$ 1 , where $M$ and $R$ are the total mass and initial radius of the cluster. For example, if the total mass of the cluster is $M=$ $1000 M_{\odot}$ and the initial radius is $R=1 \mathrm{pc}$, then one time unit is $\approx 1.5 \mathrm{Myr}$ (see Heggie \& Mathieu 1986 for details of $N$-body units).

Figure 2 shows that the level of substructure tends to decrease with time, and all substructure has essentially disappeared by $T=3$. An interesting feature is the transient rise in the level of substructure in clusters with high $D$ (i.e. those with initially low levels of substructure). This is due to the coherence in the initial velocity field. Even though there is initially a low 

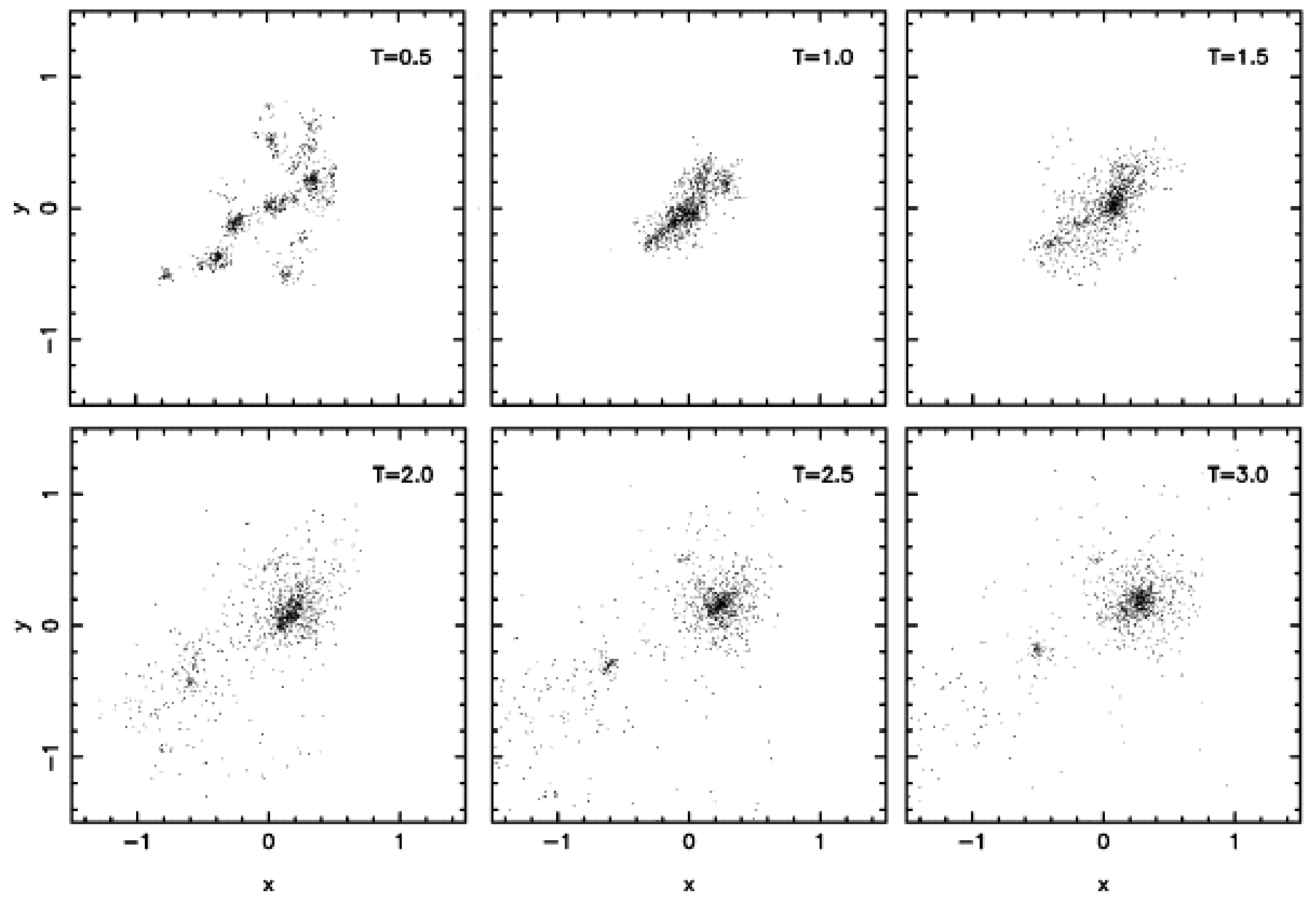

Fig. 3. The evolution of an initially $F_{\mathrm{dim}}=2$ cluster with $N=1000$ and a virial ratio $\alpha=0.1$ with coherent velocities. The time in $N$-body units is given in the top right of each panel.

level of density substructure, for a short time the coherent velocity field generates substructure. However, this substructure does not last long.

The main mechanism for erasing substructure is the gravitational interactions between clumps. The potential of a clumpy cluster is highly uneven and violent relaxation occurs, allowing the stars to relax into a smooth distribution on a short timescale. Two-body encounters also act to remove kinetic energy from the main cluster by ejecting stars. This can be seen in a rapidly expanding halo of stars around the main cluster, and a cluster core which is often significantly smaller than the initial size of the system.

Figure 3 shows in detail the evolution of the $D=2$ cluster from the previous figure. By inspection the evolution of the substructure is well described by our measures $F_{20}$ and $F_{50}$. In Fig. 3 the initially very clumpy distribution rapidly collapses. At first some of the clumps disperse, but those which are bound collapse and the larger of these clumps then attract nearby clumps. The initial clumpiness is erased as most clumps merge. However, one of the merging clumps is sufficiently bound to survive the initial merger process and emerges at late times to the bottom left. The re-emergence of this clump explains the increase in $F_{20}$ and $F_{50}$ for this cluster in Fig. 2 around $T=2$.

\subsection{Virialised clusters}

Figure 4 shows the evolution of $F_{20}$ and $F_{50}$, as in Fig. 2, but for clusters with an initial virial ratio of $\alpha=0.5$. Clusters with $\alpha=0.5$ have enough kinetic energy to support themselves against overall collapse, but their clumpy nature means that significant dynamical evolution still occurs. The most significant features of Fig. 4 are the longer time required for substructure to be erased, and the persistence of substructure throughout the simulation in the $D=1.6$ cluster.

The initial coherence of the velocity dispersion in the high- $D$ simulations is again responsible for the increase in substructure early on, although this substructure is erased over a timescale of a few time units (a few Myr in a typical cluster).

In the $D=1.6$ cluster the survival of substructure is due to the high velocity dispersion and its coherence. As can be seen in Fig. 5 the initial cluster rapidly divides into a main cluster and a lumpy sub-cluster, which has sufficient bulk velocity to escape from the main sub-cluster, dividing into 3 small subsub-clusters as it does so.

Such an evolution is typical for clusters of low fractal dimension and not unusual for clusters of higher fractal dimension. The key is the coherent nature of the velocity dispersion. The velocity dispersion within a sub-cluster holds it up against 

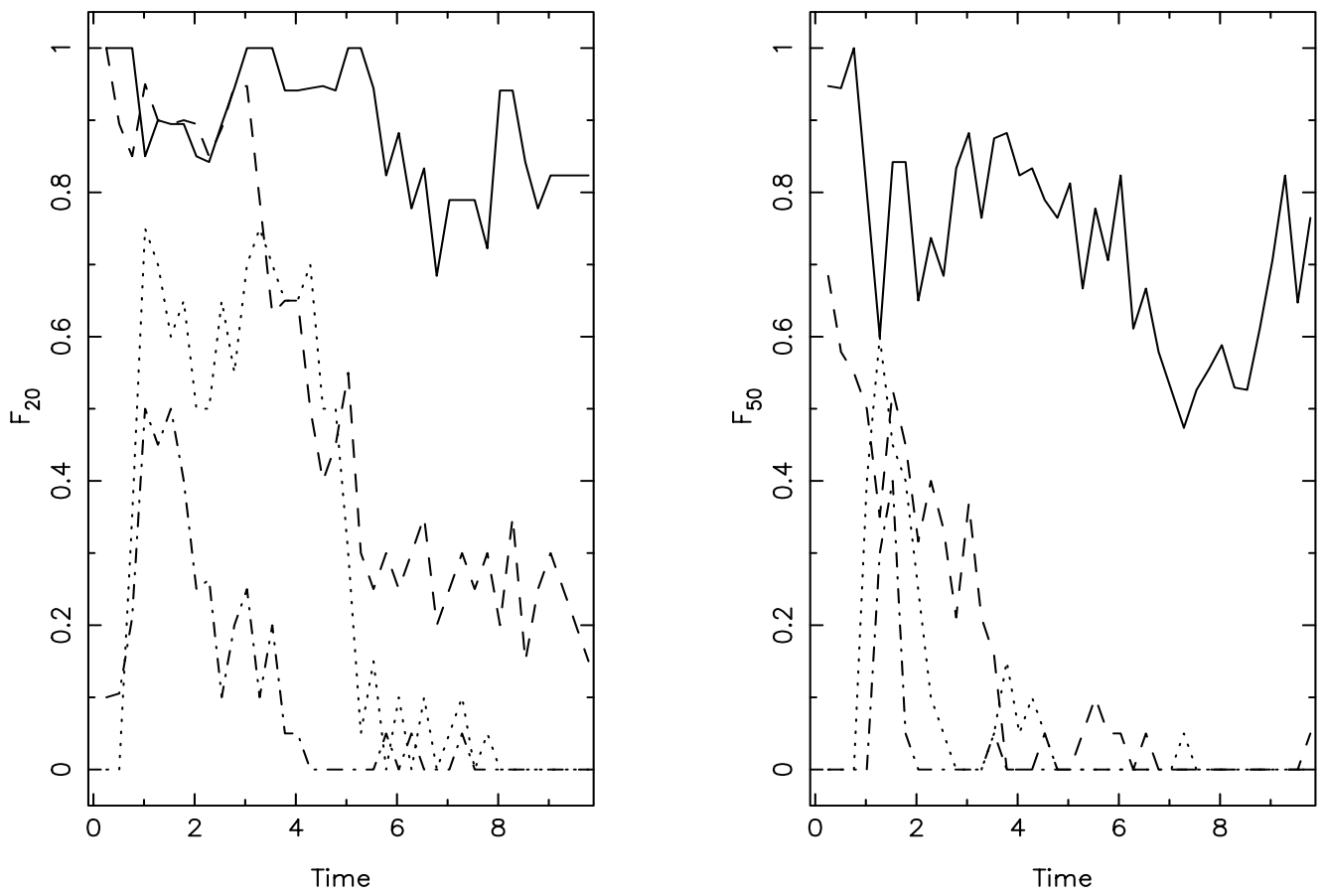

Fig. 4. The same as Fig. 2 but for clusters with an initial virial ratio of $\alpha=0.5$.
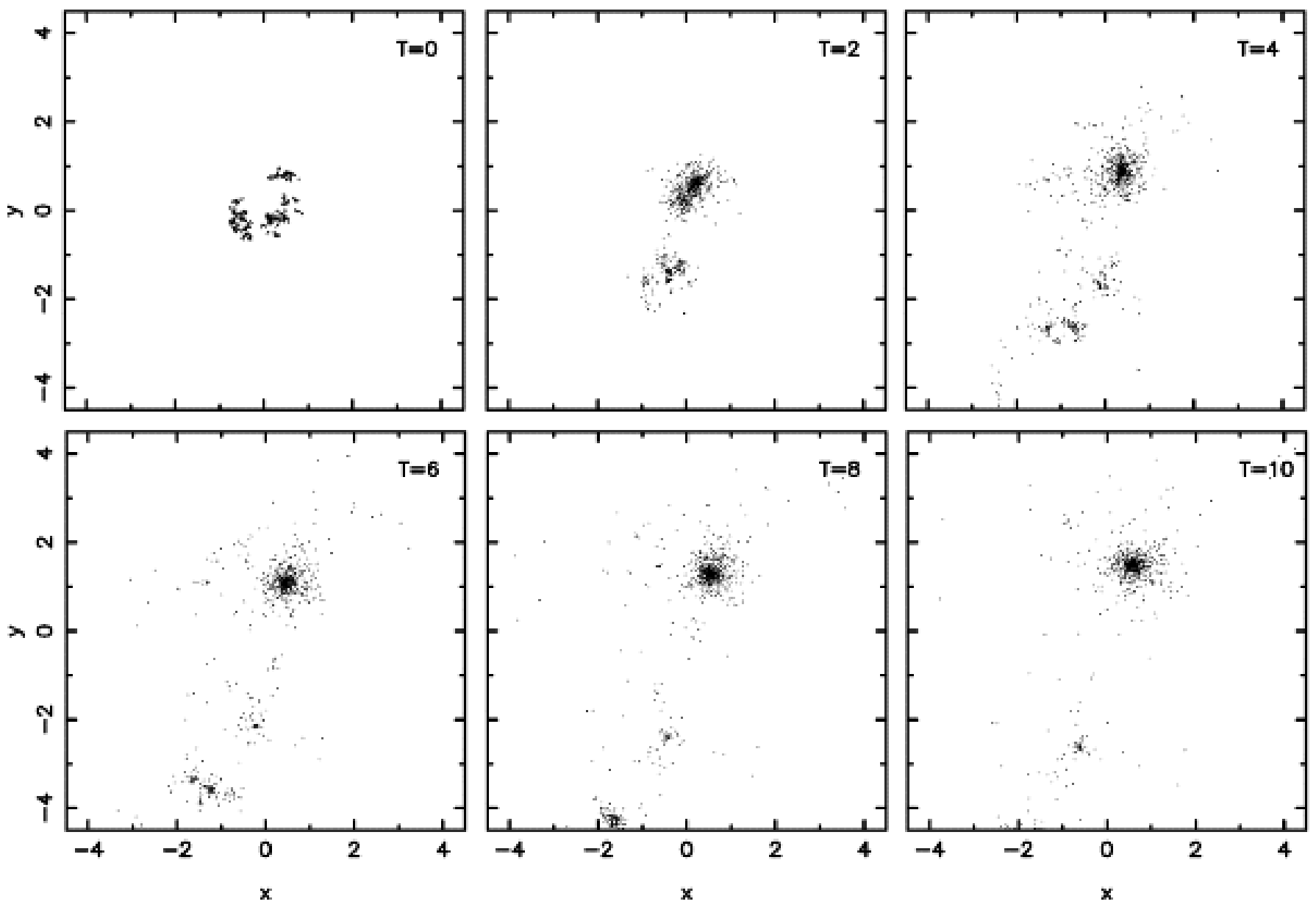

Fig. 5. The same as Fig. 3 but for a cluster with an initial virial ratio of $\alpha=0.5$. 


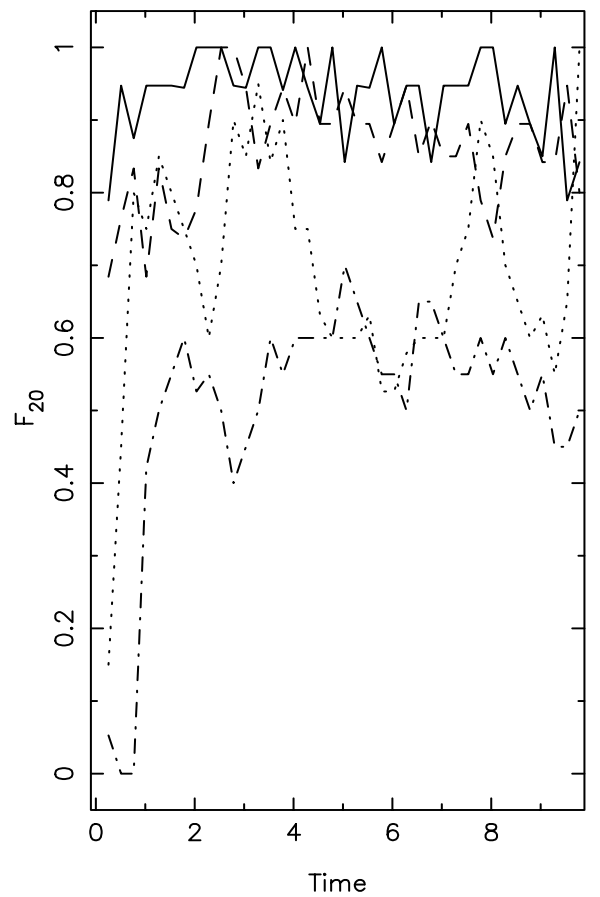

Fig. 6. As Fig. 2 but for clusters with an initial virial ratio of $\alpha=0.75$.

collapse, and its bulk velocity helps it to avoid merging with other sub-clusters. Hence it is able to maintain its separate identity for a long time. This contrasts with the cases discussed in the previous subsection, where the collapse tends to erase substructure rather quickly.

\subsection{Supervirial clusters}

Figure 6 again shows $F_{20}$ and $F_{50}$, this time for clusters with an initial virial ratio of $\alpha=0.75$, such that they expand. In these cases it is clear that the level of substructure does not decrease rapidly. Significant substructure remains at the end of the simulations for all $D$.

Even clusters whose density structure is initially not very clumpy grow density substructure, again due to the coherence of their initial velocity dispersion. Generally, the lower $D$, the more clumps there are, and the smaller they are. For high $D$ often a binary cluster is formed with two major sub-clusters. Figure 7 shows the final states (at $T=5$ ) for different realisations of clusters with a variety of initial fractal dimensions. In virtually all simulations clusters still have a significant amount of well defined substructure present at $T=5$. (For total mass $M \approx 1000 M_{\odot}$ and initial radius $R \approx 1 \mathrm{pc}, T=5$ corresponds to 5 to $10 \mathrm{Myr}$.)

Typically, by $T=5$ a main sub-cluster is identifiable (as a sub-cluster that is significantly larger than all other subclusters). Figure 7 shows in most cases a clear main sub-cluster with surrounding substructure. In many cases the substructure is never erased altogether; some clumps are not bound to the main clump and so escape. This is very common in clusters with an initial virial ratio of $\alpha=0.75$, and even occurs occasionally in clusters with an initial virial ratio of $\alpha=0.5$. Sub-clusters that are bound to the main sub-cluster can remain as separate entities for a significant length of time. We ran a

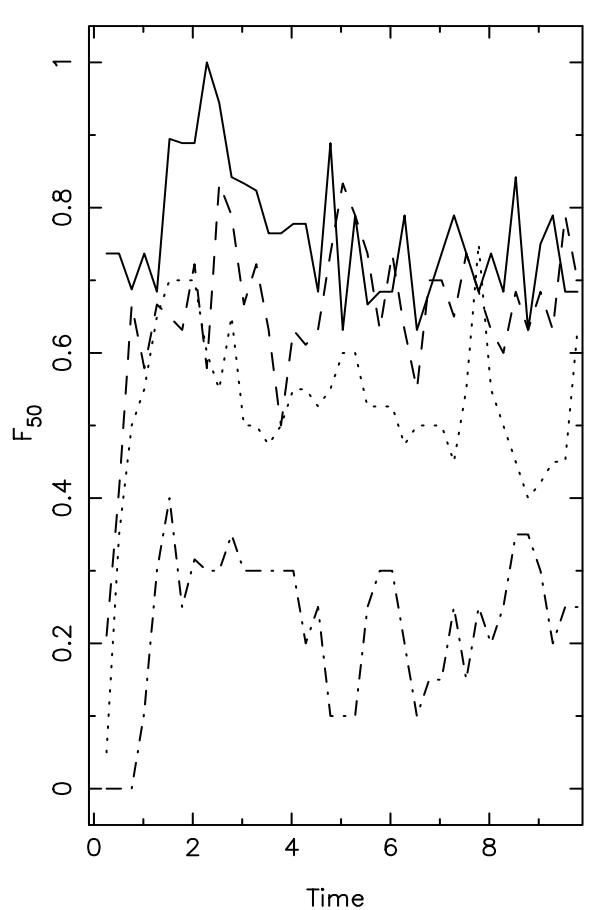

subset of our simulations until $T=100$ and found some subclusters remaining in orbit. However, in the majority of cases the tidal effect of the main sub-cluster disrupts any bound substructure by $T=10$ or 20 (roughly 10 to $50 \mathrm{Myr}$ ). This leaves a smooth, but typically elliptical, main cluster surrounded by a large, and expanding, halo of stars. We did not however investigate the details of this phase of cluster evolution in any detail.

\subsection{Incoherent velocity structures}

Given the potential importance of coherent velocity dispersion in maintaining - and even increasing - the level of substructure, it is expected that clusters with incoherent velocity dispersion will not retain substructure so long. Figure 8 shows the evolution of $F_{20}$ and $F_{50}$ for a cluster with initial virial ratio $\alpha=0.75$ and incoherent velocity dispersion. The substructure is erased almost immediately. This is unsurprising, as, with such a large virial ratio, any density substructure almost instantly disperses.

However, we believe that coherent velocity dispersion is the more plausible initial condition. The likely cause of the substructure that is commonly observed in young star clusters is the formation of sub-clusters of stars in distinct molecular cores formed by the turbulence in the molecular cloud. It is therefore to be expected that the stars within a sub-cluster have similar velocities, and that the velocity dispersion is correlated with the density substructure.

\subsection{The effect of large $N$}

The previous results have concentrated on clusters with $N_{\text {tot }}=$ 1000 representing moderately rich, open clusters (for example, the Pleiades or Orion). Here we investigate the effect of larger $N_{\text {tot }}$ on the survival or destruction of substructure. 


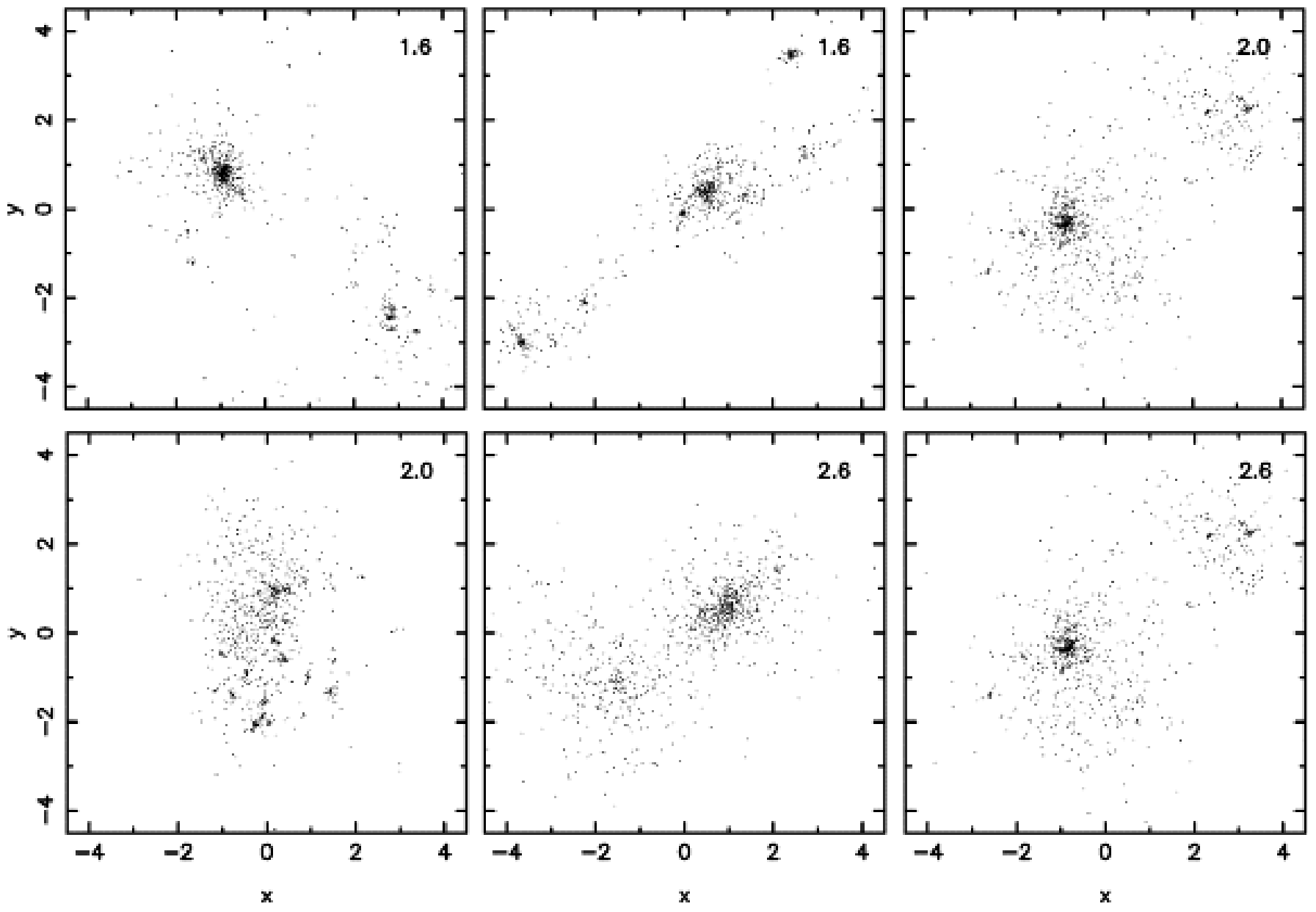

Fig. 7. The final states of different realisations of clusters at $T=5$, with $N=1000$, virial ratios of $\alpha=0.75$ and coherent velocities. The initial fractal dimension is labeled in each case in the top right.


Fig. 8. As Fig. 2 but for clusters with an initial virial ratio of $\alpha=0.75$ and a random rather than coherent velocity substructure. 

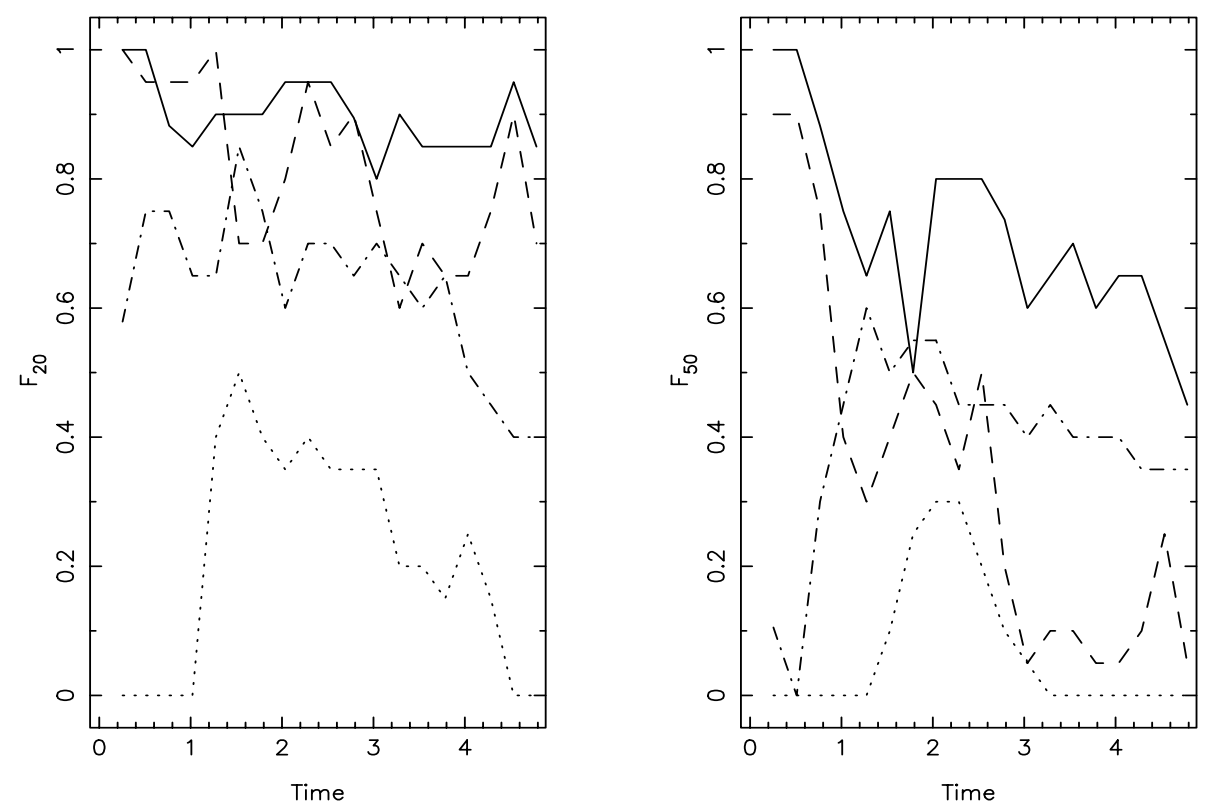

Fig. 9. As Fig. 6 but for clusters with $N=10000$.

Figure 9 shows the evolution of $F_{20}$ and $F_{50}$ for clusters with virial ratio $\alpha=0.75$, coherent velocity dispersion, and $D=1.6,2,2.6$, and 3 , equivalent to Fig. 6 but with $N_{\text {tot }}=$ 10000 . Figure 9 illustrates a common theme for large $N_{\text {tot }}$, namely that the decline in $F_{20}$ and $F_{50}$ is somewhat more rapid than in clusters with small $N_{\text {tot }}$.

This result is largely an artifact of the clump detection proceedure. Often in $N=1000$ clusters the number of particles within a particular radial bin is small (of order a few), whilst in $N=10000$ clusters that number is (obviously) far larger. Thus poisson noise has more of an effect upon the statistics in low- $N$ clusters.

As an example, two $\alpha=0.75, D=2$ clusters were simulated, one with $N=10000$ and the other with 90 per cent of the particles from the first simulation removed at random. At $T=5$, the $N=1000$ cluster had $F_{20}=0.85$ and $F_{50}=0.25$, and for $N=10000, F_{20}=0.70$ and $F_{50}=0.05$. The $F$-statistics in the $N=1000$ simulation were increased by the effect of low numbers of particles (5 to 10) in the inner radial bins, while the $N=10000$ cluster suffered far less from this effect with no less than 20 particles in any one bin. The density of particles in low- $N$ simulations is also less smooth than in high- $N$ simulations as the search radius in which 5 particles are to be found is usually significantly greater. Small clumps in low- $N$ simulations tend to be more above the average density than in large- $N$ simulations.

Nonetheless, large $N_{\text {tot }}$ clusters still maintain significant levels of substructure for several crossing times. This may be an explanation for some of the anomalous bumps observed in the profiles of several LMC star clusters (e.g. Mackey \& Gilmore 2003).

\subsection{The final structure of clusters}

An examination of the most significant sub-clusters at the end of simulations shows that they generally appear similar to older clusters. The relaxation of the clusters creates Plummer- or
King-like profiles, i.e. a flat central density profile, with a steep decline in the halo. This is especially clear in clusters with $N=10000$. The cluster illustrated in Fig. 5f, has a flat central surface density of $\approx 500$ stars per unit area over a radius of $\approx 0.5$, followed by an approximate $r^{-2}$ decline. There are significant sub-clusters apparent in the halo, the largest of which cause "bumps" to appear in the surface density profile. For clusters with low $N$, the trend to form core-halo density structures is present, but far less clear due to low- $N$ noise.

Some clusters have distinct, unbound sub-clusters which are included in our clumpiness determinations. Once a subcluster has travelled a significant distance from the main cluster it will be difficult (without proper motions) to determine that it formed in the same position as the main cluster. Thus it may appear as if they are smooth and separate clusters that formed coevally, rather than the result of dynamical segregation from the same initial cluster.

\section{Implications for observations and simulations}

Observations of young star clusters often show a very inhomogeneous, clumpy distribution. Our simulations demonstrate that if the velocity dispersion of these clusters is low, then much of that initial substructure will be erased in the ensuing collapse (cf. Aarseth \& Hills 1972). However, if, as we might expect, the velocity dispersion is high, such that the cluster remains supported or even expands, then significant levels of substructure can survive for several crossing times. Even an initially homogeneous distribution of stars can grow substructure if the initial velocity dispersion is coherent.

These results have two important consequences. First, young clusters probably undergo significant and rapid dynamical evolution. Therefore drawing conclusions about the initial stellar distribution from observations is very difficult, and should take these considerations into account. Taurus is a good example of a young, embedded cluster with a high level of 
substructure (Briceno et al. 1993; Ghez 1993). Our results imply that, when Taurus expels its residual gas and becomes a pure $N$-body system, it will probably not simply collapse into a small, dense open cluster. Indeed, it is more likely that it will separate into two or three small clusters, which in a few Myrs may look as though they formed separately from each other. As we have shown, this depends crucially on the virial ratio of the final (gas free) cluster, and on the coherence of the velocity dispersion. Even a cluster which is initally fairly smooth in appearance, such as IC348 (Najita et al. 2000), may develop substructure and look very different in a few Myrs.

Second, the rapid dynamical evolution early in a cluster's life is also important when setting the initial conditions for $\mathrm{N}$-body simulations of cluster evolution. Simple initial conditions (such as Plummer spheres) will fail to capture this potentially important stage of cluster evolution. Processes like binary capture or dissolution, and stellar ejection, which probably occur in the first few Myr of a cluster's lifetime, may be strongly affected by the evolving density substructure, and the dynamical changes we have identified here.

Acknowledgements. The GRAPE-5 was purchased on PPARC grant PPA/G/S/1998/00642, SPG is supported by PPARC grant PPA/G/S/1998/00623.

\section{References}

Aarseth, S. J. 2000, in The Chaotic Universe, ed. V. G. Gurzadyan, \& R. Ruffini (World Scientific), 286

Aarseth, S. J., \& Hills, J. G. 1972, A\&A, 21, 255

Bate, M. R., Clarke, C. J., \& McCaughrean, M. J. 1998, MNRAS, 297, 1163

Briceno, C., Calvet, N., Gomez, M., et al. 1993, PASP, 105, 686

Ghez, A. M., Neugebauer, G., \& Matthews, K. 1993, AJ, 106, 2005

Gladwin, P. P., Kitsionas, S., Boffin, H. M. J., \& Whitworth, A. P. 1999, MNRAS, 302, 305

Goodwin, S. P. 1997, MNRAS, 284, 785

Goodwin, S. P. 1998, MNRAS, 294, 47

Heggie, D. C., \& Mathieu, R. D. 1986, in The use of supercomputers in stellar dynamics, ed. P. Hut, \& S. McMillan (Springer-Verlag), 233

Hurley, J., Tout, C. A., Aarseth, S., \& Pols, O. R. 2001, MNRAS, 323, 630

Kroupa, P., Aarseth, S., \& Hurley, J. 2001, MNRAS, 321, 699

Kawai, A., Fukushige, T., Makino, J., \& Taiji, M. 2000, PASJ, 52, 659

Mackey, A. D., \& Gilmore, G. F. 2003, MNRAS, 338, 85

Najita, J. R., Tiede, G. P., \& Carr, J. S. 2000, ApJ, 541, 977

Portegies Zwart, S. F., McMillan, S. L. W., Hut, P., \& Makino, J. 2001, MNRAS, 321, 199

Williams, J. 1999, in Interstellar turbulence, ed. J. Franco, \& A. Carraminana (CUP), 190 